\title{
Random Brownian Motion Regulates the Quantity of Human Immunodeficiency Virus Type-1 (HIV-1) Attachment and Infection to Target Cell
}

\author{
Akira Tempaku* \\ School of Medicine, Faculty of Medical Sciences, University of Fukui, 23-3 Shimoaizuki, Matsuoka-cho, Yoshida-gun, Fukui 910- \\ 1193, Japan
}

(Received November 22, 2004; Accepted December 21, 2004; Published online December 24, 2004)

\begin{abstract}
The efficiency of human immunodeficiency virus type-1 (HIV-1) infection in GHOST/CXCR4 cell was controlled by the frequency of virus access, which was limited by random Brownian motion. Prolonged exposure of cell to virus increased the number of infection. Virus migrated to cell, continuously, during exposure period. Virus adsorption amount increased kinetically. It was confirmed by measurement of accumulated provirus DNA sum that the amount of virus entry depended on exposure time. Increment of virus adsorption amount and the number of infection followed to the theoretical manner that virus adsorption dynamics was regulated by random Brownian motion. This observation says that infectivity of lentivirus vector (HIV-1 based) increases by exposure time dependent way. This phenomenon is applicable to enhancing the efficiency of gene transduction by lentivirus vector.
\end{abstract}

Key words — HIV-1, adsorption, kinetic

\section{INTRODUCTION}

Virus replication process includes many steps. Mechanism of virus adsorption on cell surface has well studied. ${ }^{1-8)}$ Prior to cell-virus association, virus migrates toward target cell. During this period, random Brownian motion regulates virus particle (virion) movement. ${ }^{9)}$ It was reported that artificially increased virus-cell contact chances by flow-through infection procedure enhanced virus infection. ${ }^{9)}$ That

*To whom correspondence should be addressed: School of Medicine, Faculty of Medical Sciences, University of Fukui, 23-3 Shimoaizuki, Matsuoka-cho, Yoshida-gun, Fukui 9101193, Japan. Tel.: +81-776-61-3111; Fax: +81-776-61-8153; E-mail: ST04057@fmsrsa.fukui-med.ac.jp observation informs to us the efficiency of virus infection is regulated by virion dynamics.

Lentiviruses have attracted much attention as gene transfer vehicles. Different from other vectors, exogene mediated by lentivirus vector can be kept stably during cell proliferation. Furthermore, lentivirus vector can introduce gene into non-dividing cell, while retrovirus vector has some difficulty. However, lentivirus vector has a disadvantage of low gene transduction efficiency. Developing new strategies with high efficiency of gene transduction has been strongly desired. Genetic modifications of vector virus to overcome several restrictions during replication cycle in host cell have been studied. In contrast, modulation of cell-virus association frequency has been less focused, except studies using chemical substances. ${ }^{10,11)}$

Virus infection initiates with direct contact between cell and virus, as a consequence of cell and virus collision. Enhancing the frequency of virion attachment to target cells is a solution to increase gene transduction efficiency. From the evidence in previous report, ${ }^{9)}$ this research aims to clear the relationship between the efficiency of virus infection and virion dynamics. Virion diameter is similar to colloidal particle $(100 \mu \mathrm{m})$. It was predicted that random Brownian motion of virion might have an important role during the flowing in medium. This study focuses to reveal whether random Brownian motion of virion regulates the efficiency of virion access to cell and attachment on cell surface.

\section{MATERIALS AND METHODS}

Cell and Pseudotyped Viruses GHOST/

CXCR4 cell (developed from human osteosarcoma cell; HOS) was maintained in Dulbecco's modified 
Eargle's medium (ICN, Costa Mesa, CA, U.S.A.) supplied with $10 \%$ of heat inactivated fetal calf serum (PAA, Linz, Austria), streptomycin $(100 \mu \mathrm{g} / \mathrm{ml})$, penicillin $(100 \mathrm{IU} / \mathrm{ml})$, geneticin $(500 \mu \mathrm{g} / \mathrm{ml})$, Hygromycin B $(100 \mu \mathrm{g} / \mathrm{ml})$ and puromycin $(50 \mu \mathrm{g} / \mathrm{ml})$. Luciferase carrying pseudotyped virus was made by transfection of HIV-1 molecular clone vector: pNL4-3-luc ${ }^{12}$ with NL4-3 envelope expression vector (pCXN NL4-3) into 293T cell by calcium phosphate precipitation method. Enhanced green fluorescence protein (EGFP) carrying pseudotyped virus was developed by inserting EGFP gene (obtained from pEGFP-N1; Clontech, Palo Alto, CA, U.S.A.) into XhoI to NotI site in pNL4-3luc. Modified construct was co-transfected with NL4-3 envelope expression vector.

Assessment of Virus Infection Kinetics - NL43 enveloped pseudotyped virus (carrying luciferase reporter gene) infected GHOST/CXCR4 cell $(2 \times$ $10^{4}$ cells in 48 -wells plate). Leu3a (anti CD4 antibody; Pharmingen, San Diego, CA, U.S.A.) or 12G5 (anti CXCR4; Pharmingen) were sequentially (0.5, $1,2,4,8,12$ and $24 \mathrm{hr}$ post virus inoculation) added to virus (final $10 \mu \mathrm{g} / \mathrm{ml}$ ). As a mock test, MOPC-21 (Cappel, Aurora, Ohio, U.S.A.) antibody was added. Twenty-five hours post infection, all cells were washed by medium. Three days later, virus infectivity was measured by luciferase assay following the manufacture's instruction (Promega, Madison, WI, U.S.A.).

Luciferase Assay — Seventy-two hours post virus infection, cell was washed with PBS and lysed with $100 \mu$ of $1 \times$ lysis buffer (Promega). Luciferase activity was measured after mixing $10 \mu$ of lysate with $50 \mu \mathrm{l}$ of luciferase substrate solution (Promega) by counting the light intensity with luminometer (Lumat LB 9501/16; EG\&G Berthold, Bad Wildbad, Germany). All tests were performed as duplicate.

Measurement of HIV-1 Core Antigen (p24) Amount — NL4-3 virus infected GHOST/ CXCR 4 cells $\left(2 \times 10^{4}\right.$ cells in 48 -wells plate). Thirty minutes, 1, 2, 4, 8, 12 and $24 \mathrm{hr}$ after inoculation, cell associated virus (absorbed) quantity and cell free virus (in culture supernatant) quantity (HIV-1 core antigen p24 amount) were measured by ELISA using RETRO-TEK HIV-1 p24 antigen ELISA system (ZeptoMetrix, Buffalo, NY, U.S.A.). Virus containing supernatant was subjected to cell free virus specimen. Remained cell was rinsed with medium twice. Then, cell was lysed with lysis buffer (RETRO-TEK HIV-1 p24 antigen ELISA system) and subjected to cell associated virus specimen.
Polymerase Chain Reaction (PCR) - NL4-3 virus infected GHOST/CXCR4 cell $\left(5 \times 10^{5}\right.$ cells in 6-wells plate). One or twelve hours later, amount of entered virus was quantified by PCR. Briefly, cell was lysed with urea lysis buffer (4.7 M urea, 1.3\% SDS, $0.23 \mathrm{M} \mathrm{NaCl}, 0.67 \mathrm{mM}$ EDTA, $6.7 \mathrm{mM}$ Tris pH 8.0). DNA was isolated by phenol chloroform extraction method followed by ethanol precipitation. PCR was performed in $1 \times$ PCR buffer $(20 \mathrm{mM}$ Tris pH 8.0, $50 \mathrm{mM} \mathrm{KCl}, 1.5 \mathrm{mM} \mathrm{MgCl}, 0.2 \mathrm{mM}$ each dNTPs and $0.05 \mathrm{U} / \mu \mathrm{l}$ recombinant Taq DNA polymerase) with each $0.5 \mathrm{mM}$ of M667 (5'-GGCTA ACTAGGGAACCCACTG-3') and M661 (5'CCTGCGTCGAGAGAGCTCTGGTTT-3') primers to amplify provirus DNA (R/gag region), or glo-A (5'-ACACAACTGTGTTCACTAGC-3') and glo-B (5'-CAACTTCATCCACGTTCACC-3') primers to amplify $\beta$-globin coding DNA. ${ }^{13)}$

FACScan — EGFP reporter gene carrying NL43 enveloped pseudotyped virus (150 ng) infected GHOST/CXCR 4 cell $\left(8 \times 10^{4}\right.$ cells in 24 -wells plate). Exposing virus was removed 0.5, 1, 2, 4, 8, 12 or $24 \mathrm{hr}$ later. Two days post virus infection, EGFP positive cell ratio (in $1 \times 10^{4}$ cells) was evaluated by FACScan (Beckton Dickinson Immunocytometry System, San Jose, Calif., U.S.A.).

\section{RESULTS AND DISCUSSION}

\section{Exposure Time Dependent Increment of HIV-1 Infection}

Number of HIV-1 infection events increased doubly for early $8 \mathrm{hr}$ (Figs. 1 and 2). About ten times higher amount of virus infected cell after $12 \mathrm{hr}$ exposure than $1 \mathrm{hr}$ one (Fig. 1). Result of Fig. 1 meant antibodies against receptors for HIV-1 blocked adsorption of slowly migrating virus toward receptors. It said that virus adsorption to cell surface receptor gradually occurs. These results were confirmed by three times experiments and a representative result is showed (Fig. 1).

Changing target cell number $\left(1-6 \times 10^{4}\right.$ cells per well of 48-wells plate) had almost no effect to total infection counts as previously described ${ }^{14)}$ and it did not affect to increasing infectivity (data not shown).

Concentration of virus in medium was critical factor to determine the final sum of infection. However, each concentration case (2-20 ng p24 per ml), maximum point of infectivity was detected at 12 to $24 \mathrm{hr}$ later (data not shown). Highly concentrated virus (20 ng) marked high infectivity than lower 


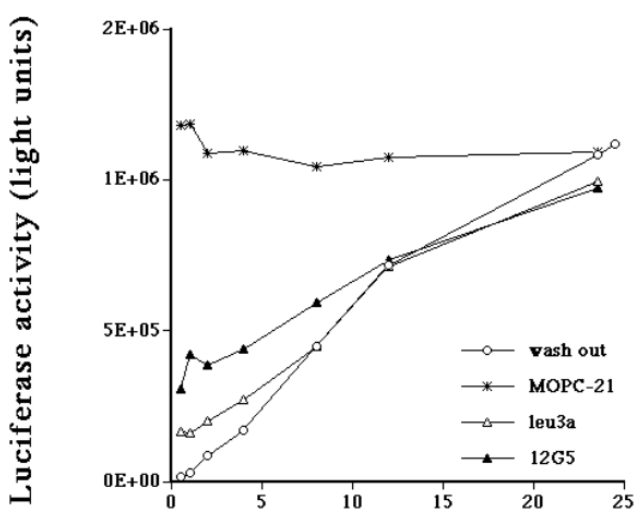

Time post infection (h)

Fig. 1. Virus Infection Occurs for Prolonged Exposure Time Long

Treatments of virus solution; wash out (open circle), add leu3a (open triangle), add 12G5 (solid triangle) and add MOPC-21 (asterisk).

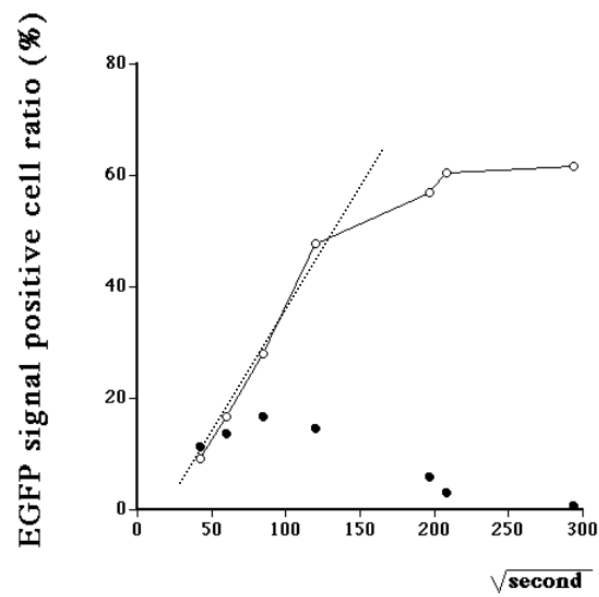

Fig. 2. Exposing Virus was Removed 0.5, 1, 2, 4, 8, 12 or $24 \mathrm{hr}$ Later (Open Circle Line)

Solid circle show that NL4-3 pseudotyped virus infected GHOST/ CXCR4 cell, after $0.5,1,2,4,8,12$ or $24 \mathrm{hr}$ incubation at $37^{\circ} \mathrm{C}$ in cell free condition. Virus was exposed to cell for $1 \mathrm{hr}$ at $37^{\circ} \mathrm{C}$ and was washed. Dot plots line means the theoretical line: if infection events completely obeyed to random Brownian motion theory.

concentration virus ( $2 \mathrm{ng}$ ) (data not shown), as already reported. ${ }^{14)}$ The former case showed sharp curve of increment, during the latter marked moderate tendency of it. High concentration virus showed high count of infectivity than by lowers one at each interval of exposure time. In all cases, the maximal point of infectivity came after $12 \mathrm{hr}$ long exposure (data not shown). Furthermore, same virus concentration showed similar infection-increment curve, even so total volume of virus solution was varied (data not shown).

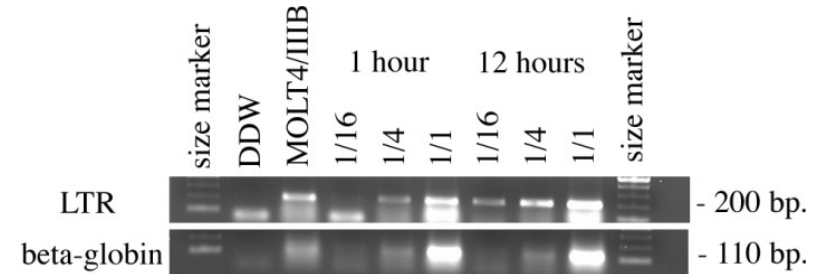

Fig. 3. Entered Virus Amount is Due to Adsorption Time Long Provirus DNA amount carried by 1 or $12 \mathrm{hr}$ long virus-exposing cell was measured by semi-quantitative PCR. Sequentially diluted (1/1, $1 / 4$ and 1/16) DNA was subjected to. Size marker lanes meant $100 \mathrm{bp}$. ladder marker DNA loading. DDW lane was negative control of PCR. MOLT4/IIIB lane was positive control of PCR for provirus DNA.

This experiment used replication incompetent pseudotyped virus. Thus, it was possible to examine a single replication cycle progress without complication of multiple effect in replication. The limiting factor of peak infectivity count was not absolute amount of virion, but density of virion, which regulates frequency of virus-receptor interaction. These results confirmed that the frequency of virus access toward cell (receptor on cell surface) limits virus infectivity. High-density virion containing solution has much potential of virus-cell interaction. Furthermore, long time exposure to virus also increases chance of virus-cell interaction.

Supporting virus attachment on cell surface by polybrene enhanced total infection mass (data not shown) as previously described. ${ }^{11)}$ Long time exposure of cell to virus with polybrene also up-regulated infectivity at all time points (data not shown). Whether virus infection was tried with or without polybrene, maximum infectivity was monitored at same time (about $12 \mathrm{hr}$ post infection). Nonetheless the slope of infectivity curve inclined sharply by addition of polybrene, all infection completed after $12 \mathrm{hr}$ exposure (data not shown). It said that the increment of virus infection was due to the frequency of virus access to cell. In other word, enhancement of virus infectivity with polybrene, kinetically, occurred before virion attachment to cell surface.

\section{Accumulation of Virus Attachment and Entry}

Twelve hours long exposure of virus up-regulated the sum of entry than that of 1-hr case. Increased adsorption and penetration of virus resulted in large amount of provirus DNA accumulation (Fig. 3). FACS analysis showed the number of virus infecting cells increased kinetically (Fig. 2). This increment of infection was due to the increment of virus adsorption amount (p24) (Fig. 4) and entry sum 


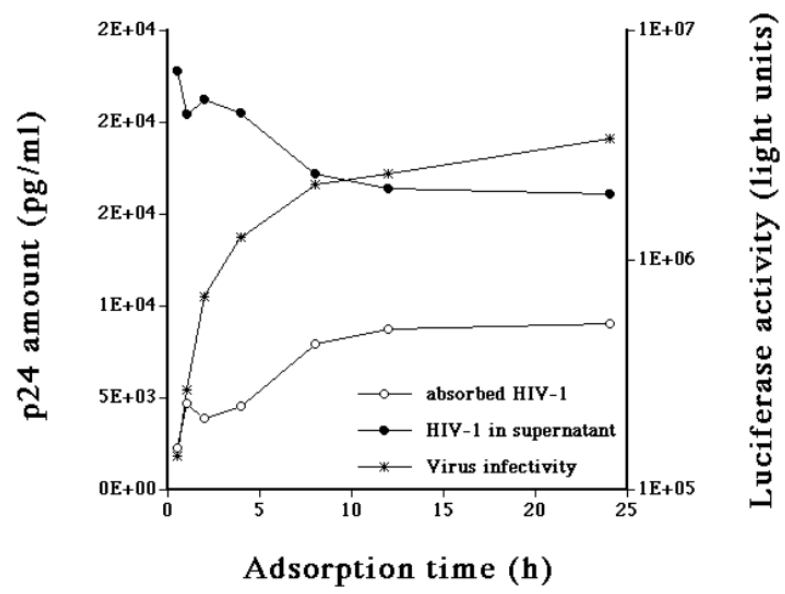

Fig. 4. Virus Adsorption Occurs during Prolonged Exposure Period

Cell associated HIV-1 core antigen (p24) amount (open circle) and cell free p24 mass (solid circle) were monitored, kinetically. HIV-1 infection (asterisk) was aligned by right side vertical plot scale.

(provirus) (Fig. 3). Adsorption of virus on cell increased doubly for initial 4 to $8 \mathrm{hr}$ (Fig. 4). Cell free virus amount decreased during adsorption period, in anti-parallel (Fig. 4). Addition of antibodies against CD4 or CXCR4 to inoculating virus, kinetically, had same effect of washing out virus (Fig. 1).

\section{Random Brownian Motion Limits Virus Dynam- ics}

Increment of virus infection was due to random Brownian motion theory (Fig. 2). The dynamics of virus infecting cell number was almost similar to the theoretical way, ${ }^{3,4)}$ which should be marked if random Brownian motion controls all particles movement, perfectly. For early $4 \mathrm{hr}$, virus had enough competency of infection to cell (solid circles in Fig. 2). The infectivity curve (open circle line in Fig. 2) was overlapped to the theoretical line (dot plot line in Fig. 2).

Virus infectivity for 1-hr exposure decreased during late period. More than $8 \mathrm{hr}$ incubation of virus at $37^{\circ} \mathrm{C}$ reduced the competency of infection. Virus infectivity was diminished from $15 \%$ of cell per $1 \mathrm{hr}$ (during early $4 \mathrm{hr}$ ) to less than $5 \%$ of cell per $1 \mathrm{hr}$ (later than $8 \mathrm{hr}$ ). The plateau period of virus infectivity dynamics was due to the inactivation of virion by incubation at $37^{\circ} \mathrm{C}$ (Fig. 2).

This study revealed random Brownian motion controls virion movement. Exposure time dependent up-regulation of virus infectivity was not only NL43 (X4 HIV-1) to GHOST/CXCR4 cell case. Infection of other virus strains (JR-FL; R5 HIV-1, amphotropic murine leukemia virus: A-MuLV, vesicular stomatitis virus: VSV) to adequate cells (GHOST/CCR5, MAGI/CCR5, U937, MOLT-4, peripheral blood mononuclear cell or macrophage) was also increased kinetically (data not shown). Special effect of target cell type was not observed among all cells used. From adherent cells to floating cells, native cells and immortalized cells, all cells had the increment of virus infectivity, kinetically. Non-dividing cell (macrophage) also showed the increment of virus (JR-FL and VSV) infectivity (data not shown). These results support the theory that random Brownian motion controls virion migration to cell. Long time exposure of cell to virus resulted in accumulation of virus attachment on cell surface and penetration to cell.

This evidence is useful knowledge to apply gene transfer method using lentivirus vector. Especially, vector with A-MuLV envelope is already used for several clinical trials as a vehicle of gene. Observation in this research proposes much efficient way of gene transduction to target cell. Simply, long time exposure of cell to vector virus up-regulates virus infection and gene transduction efficiencies.

Acknowledgements This work was supported by grants from the Ministry of Education, Science, and Culture, and the Ministry of Health and Welfare, Japan.

\section{REFERENCES}

1) Puck, T. T., Garen, A. and Cline, J. (1951) The mechanism of virus attachment to host cells, I. The role of ions in the primary reaction. J. Exp. Med., 93, 65-88.

2) Valentine, R. C. and Allison, A. C. (1959) Virus particle adsorption, I. Theory of adsorption and expression on the attachment of particles to non-biological surfaces. Biochim. Biophys. Acta, 34, 10-23.

3) Allison, A. C. and Valentine, R. C. (1960a) Virus particle adsorption, II. Adsorption of vaccinia and plague viruses to cells in suspension. Biochim. Biophys. Acta, 40, 393-399.

4) Allison, A. C. and Valentine, R. C. (1960b) Virus particle adsorption, III. Adsorption of viruses by cell monolayers and effects of some variables on adsorption. Biochim. Biophys. Acta, 40, 400-410.

5) Christensen, J. R. (1965) The kinetics of reversible and irreversible attachment of bacteriophage $\mathrm{T} 1$. Virology, 26, 727-737.

6) Gamow, R. I. (1969) Thermodynamic treatment of 
bacteriophage T4B adsorption kinetics. J. Virol., 4, 113-115.

7) Payne, J. G. and Norrby, E. (1978) Adsorption and penetration of enveloped and naked vaccinia virus particles. J. Virol., 27, 19-27.

8) Petricevich, V. L., Palomares, L. A., Gonzalez, M. and Ramirez, O. T. (2001) Parameters that determine virus adsorption kinetics: toward the design of better infection strategies for the insect cell baculovirus expression system. Enz. Microb. Technol., 29, 52-61.

9) Chuck, A. S., Clarke, M. F. and Palsson, B. O. (1996) Retrovirus infection is limited by Brownian motion. Human Gene Therapy, 7, 1527-1534.

10) Kahn, M. L., Lee, S. W. and Dichek, D. A. (1992) Optimization of retroviral vector-mediated gene transfer into endothelial cells in vitro. Circ. Res., 71, 1508-1517.
11) Davis, H. E., Morgan, J. R. and Yarmush, M. L. (2002) Polybrene increases retrovirus gene transfer efficiency by enhancing receptor-independent virus adsorption on target cell membranes. Biophys. Chem., 97, 159-172.

12) Connor, R. I., Chen, B. K., Choe, S. and Landau, N. R. (1995) Vpr is required for efficient replication of human immunodeficiency virus type-1 in mononuclear phagocytes. Virology, 206, 935-944.

13) Zack, J. A., Arrigo, S. J., Weitsman, S. R., Go, A. S., Haislip, A. and Chen, I. S. (1990) HIV-1 entry into quiescent primary lymphocytes: molecular analysis reveals a labile, latent viral structure. Cell, 61, 213222.

14) Morgan, J. R., Ledoux, J. M., Snow, R. G., Tompkins, R. G. and Yarmush, M. L. (1995) Retrovirus infection: effect of time and target cell number. J. Virol., 69, 6994-7000. 